\title{
Production, Properties and Swelling of Composite Agar-Pectic Gel Particles in an Artificial Gastroenteric Environment
}

\author{
Elena A. Mikhailova*; Anatoly A. Shubakov, PhD \\ Institute of Physiology of Komi Science Centre of the Ural Branch of the RAS, \\ FRC Komi SC UB RAS \\ Syktyvkar, Komi Republic, the Russian Federation
}

\begin{abstract}
The purpose of the present work was to obtain and study the properties of composite calcium-agar-pectic gel particles (CaAPGPs) obtained from aqueous solutions of agar (AA) and apple pectin (AP), from aqueous solutions of agar (AA) and pectin heracleuman (HS) in the presence of $\mathrm{Ca}^{2+}$ ions $(0.34 \mathrm{M})$. The swelling of the obtained composite CaAPGPs in an artificial gastroenteric environment was also investigated.

Methods and Results: We used commercial AP AU701 (AP, Herbstreith \& Fox KG, Germany), HS isolated from the aerial part of the Sosnovskyi hogweed Heracleum sosnowskyi Manden, and food agar (AA). Spherical composite CaAPGPs were obtained from low-methyl esterified AP with a molecular weight of $406 \mathrm{kDa}$, pectin $\mathrm{HS}$ with a molecular weight $>300 \mathrm{kDa}$, and food agar (AA) in the presence of $\mathrm{Ca}^{2+}$ ions $(0.34 \mathrm{M})$ as a cross-linking agent by the method of ionotropic gelation.

It was found that dry CaAPGPs based on AP (Ca-AA-AP) have a diameter of $1.16 \pm 0.14-1.23 \pm 0.05 \mathrm{~mm}$, which was greater than the diameter of dry CaAPGPs based on HS (Ca-AA-HS) $(0.95 \pm 0.12-1.16 \pm 0.05 \mathrm{~mm})$. The density of dry CaAPGPs based on $\mathrm{AP}(\mathrm{Ca}-\mathrm{AA}-\mathrm{AP})$ with an increase in the concentration of AP in their composition from $1 \%$ to $2 \%$ increased by 1.7 times - from $0.37 \pm 0.07 \mathrm{mg} / \mathrm{mm}^{3}$ to $0.63 \pm 0.05 \mathrm{mg} / \mathrm{mm}^{3}$. Dry composite CaAPGPs based on HS (Ca-AA-HS) were denser. With an increase in the HS concentration in their composition from $1 \%$ to $2 \%$, the degree of particle density increases by $2.2-$ from $0.45 \pm 0.03 \mathrm{mg} / \mathrm{mm}^{3}$ to $0.97 \pm 0.19 \mathrm{mg} / \mathrm{mm}^{3}$.

The swelling and degradation of the obtained dry composite CaAPGPs in an artificial gastroenteric environment were studied. It was found that CaAPGPs formed from 1\% AP and 2\% AA degraded almost immediately in simulated intestinal fluid. Whereas, CaAPGPs formed from $2 \%$ AP and $1 \%$ or $2 \%$ AA completely degraded in simulated colonic fluid after 1 hour of incubation in it.

CaAPGPs formed from $1 \% \mathrm{HS}$ and $2 \% \mathrm{AA}$, and particles obtained from $2 \% \mathrm{HS}$ and $1 \% \mathrm{AA}$, remained stable in simulated intestinal fluid, and then completely degraded immediately upon entering in simulated colonic fluid. CaAPGPs, consisting of $2 \% \mathrm{HS}$ and 2\% AA, dissolve in simulated colonic fluid after 1 hour of incubation in it.(International Journal of Biomedicine. 2021;11(4):456-459.)
\end{abstract}

Key Words: apple pectin • heracleuman $\bullet$ calcium ions $\bullet$ composite agar-pectic gel particles $\bullet$ artificial gastroenteric environment

For citation: Mikhailova EA, Shubakov AA. Production, Properties and Swelling of Composite Agar-Pectic Gel Particles in an Artificial Gastroenteric Environment. International Journal of Biomedicine. 2021;11(4):456-459. doi:10.21103/Article11(4)_OA11

\section{Abbreviations}

AP, apple pectin; HS, heracleuman; CaAPGPs, calcium-agar-pectic gel particles; GIT, gastroentestinal tract; SGF, simulated gastric fluid; SIF, simulated intestinal fluid; SCF, simulated colonic fluid.

\section{Introduction}

In recent years, a surge of interest has been devoted to the research of natural polysaccharides (pectin in particular) for their wide range of biological properties and different applications in pharmacology. Pectin is a polysaccharide with a core consisting of $\alpha$-1,4-linked D-galacturonic acid and $\alpha$-1,2-L-rhamnose units in turn, as well as a large number of neutral sugars, including 
arabinose, galactose, and lesser amounts of other sugars. Pectins have a linear anionic backbone with regions having no side chains known as "smooth regions," and regions with non-ionic side chains known as "hairy regions." The structural classification of pectin includes: homogalacturonan (HG), rhamnogalacturonan I (RG-I), and substituted galacturonans such as rhamnogalacturonan II (RG-II). ${ }^{(1-3)}$

In the human diet, pectin is one of the most important sources of dietary fiber. Like other types of dietary fiber, pectin is practically not depolymerized by endogenous enzymes of the gastrointestinal tract when passing through the stomach and small intestine. However, partial degradation of pectin is possible under the physicochemical conditions of the stomach and small intestine. More or less completely pectin is fermented in the human large intestine using pectinolytic enzymes produced by symbiotic microflora. ${ }^{(2)}$

Pectins, like other non-starch polysaccharides (NSPs), are used in the treatment of inflammatory bowel disease (IBD). NSPs exhibit the potential to be promising agents for adjuvant therapy and for the prevention of IBD. ${ }^{(4)}$

The use of an artificial gastroenteric environment as a model digestive system will make it possible to study the processes of biotransformation of plant food, and in particular pectins, in a healthy and/or inflamed gastrointestinal tract. The properties of pectic-gel particles largely depend on the chemical composition and macromolecular structure of pectin polysaccharides. Swelling and degradation of pectic-gel particles in the gastrointestinal tract depend on the structural and mechanical characteristics of pectin, the concentration of pectin and the type of metal ion as a cross-linking agent in the composition of pectic-gel particles, concentration of pectinases in the large intestine, $\mathrm{pH}$, and temperature. ${ }^{(2,5)}$

Composite gels based on natural polymers allow the development of new biomaterials with new physicochemical properties that will improve their functionality. Different physicochemical characteristics of composite gel particles based on different pectins can influence their stability and swelling properties in the simulated gastric environment, the environment of the small and large intestines. Composite gel particles based on the different pectins exhibit potential applications as carrier materials in controlled release systems and particularly serve as promising systems for colon-targeted drug delivery. ${ }^{(5)}$

Previously, the properties of composite gel particles based on pectin and chitosan, ${ }^{(6)}$ pectin and alginate, ${ }^{(7)}$ pectin and k-carrageenan ${ }^{(8)}$ were obtained and studied. There is information on the preparation and properties of composite gel particles formed from two different pectins. ${ }^{(5)}$ However, there is practically no data on the preparation and properties of composite gel particles formed from pectin and polysaccharide agar.

The purpose of the present work was to obtain and study the properties of composite calcium-agar-pectic gel particles (CaAPGPs) obtained from aqueous solutions of agar (AA) and apple pectin (AP), from aqueous solutions of agar (AA) and pectin heracleuman (HS) in the presence of $\mathrm{Ca}^{2+}$ ions $(0.34$ $\mathrm{M})$. The swelling of the obtained composite CaAPGPs in an artificial gastroenteric environment was also investigated.

\section{Materials and Methods}

We used commercial AP AU701 (AP, Herbstreith \& Fox $\mathrm{KG}$, Germany), pectin heracleuman (HS) isolated from the aerial part of the Sosnovskyi hogweed Heracleum sosnowskyi Manden, ${ }^{(9)}$ and food agar (AA).

Spherical composite CaAPGPs were obtained from low-methyl esterified AP with a molecular weight of $406 \mathrm{kDa}$, pectin HS with a molecular weight $>300 \mathrm{kDa}$, and food agar (AA) in the presence of calcium ions $(0.34 \mathrm{M})$ as a crosslinking agent by the method of ionotropic gelation. ${ }^{(10)}$

Composite CaAPGPs based on AP $(1 \% \mathrm{AP}+2 \% \mathrm{AA}$, $2 \% \mathrm{AP}+2 \% \mathrm{AA}, 2 \% \mathrm{AP}+1 \% \mathrm{AA}$ ) were obtained by slowly stirring a mixture of the appropriate amount of AP $(10 \mathrm{mg}$ or $20 \mathrm{mg}$ ) and the corresponding amount of AA (10 mg or $20 \mathrm{mg})$ in distilled water $(1 \mathrm{ml})$ with a magnetic stirrer MM-5 (Russia) at heating $\left(45^{\circ} \mathrm{C}\right)$ for 4 hours until complete dissolution. Composite CaAPGPs based on HS were prepared in a similar way.

Gel particles of spherical form were prepared by dropby-drop injection of corresponding solutions of mixtures of pectins with agar from a syringe through a needle with a hole diameter of $0.6 \mathrm{~mm}$ at a distance of $4-5 \mathrm{~cm}$ in the slowly stirrred calcium chloride solution $(0.34 \mathrm{M})$ and further stirring for $30 \mathrm{~min}$ at room temperature. The resulting gel particles were then washed three times in distilled water with stirring for 5 minutes and dried for $10-14 \mathrm{~h}$ at $37^{\circ} \mathrm{C}$.

The diameter and density of CaAPGPs were determined using an optical microscope (Altami, Russia) with a camera and an image analysis program (ImageJ 1.46r program, National Institutes of Health, USA). For calibration, a linear scale was used; one pixel corresponded to $0.024 \mathrm{~mm}$.

To study the swelling of dry composite CaAPGPs under conditions simulating a gastroenteric medium, artificial gastric medium (SGF), small intestine medium (SIF), and large intestine medium (SCF) were prepared as previously described..$^{(2,6)}$

To determine swelling, the corresponding dry composite CaAPGPs (1-2 mg) were placed in Petri dishes (diameter 3.5 $\mathrm{cm})$ and subsequently incubated in $3 \mathrm{ml}$ of SGF (2 h), SIF $(4 \mathrm{~h})$, and SCF solutions with shaking in a shaker (Titramax 1000 , "Heidolph", Germany) at $100 \mathrm{rpm}$ and at $37^{\circ} \mathrm{C}$. At regular intervals, the diameter and density of the gel particles were determined as described above. The experiments were performed in triplicate. The degree of gel swelling (SD,\%) was determined by the formula: $\mathrm{SD}=\left(\mathrm{D}_{1}-\mathrm{D}_{o}\right) / \mathrm{D}_{0} \times 100 \%$, where $\mathrm{D}_{1}$ - diameter of the particles $(\mathrm{mm})$ after a certain time of incubation in the medium, $\mathrm{D}_{0}$ - initial particle diameter of the particles $(\mathrm{mm})$. $^{(11)}$

The statistical analysis was performed using the statistical software BioStat (version 4.03) and Microsoft Office Excel 2007.

\section{Results and Discussion}

The morphological (size, shape) and structuralmechanical (density) characteristics of the obtained wet and dry composite CaAPGPs were studied. 
Table 1 shows the morphological and structuralmechanical characteristics of wet composite CaAPGPs. Wet spherical composite CaAPGPs formed on the basis of AP (CaAA-AP) have a diameter in the range from $3.80 \pm 0.25 \mathrm{~mm}$ to $4.28 \pm 0.25 \mathrm{~mm}$, which is larger than the diameter of wet CaAPGPs formed on the basis of pectin heracleuman (Ca-AAHS), with a diameter from $3.27 \pm 0.15 \mathrm{~mm}$ to $3.50 \pm 0.15 \mathrm{~mm}$.

The density of wet composite CaAPGPs formed on the basis of AP (Ca-AA-AP) varies from $0.35 \pm 0.05 \mathrm{mg} /$ $\mathrm{mm}^{3}$ to $0.59 \pm 0.10 \mathrm{mg} / \mathrm{mm}^{3}$, which is lower than the density of wet agar-pectin gel particles formed on based on pectin heracleuman $(\mathrm{Ca}-\mathrm{AA}-\mathrm{HS})$, which ranges from $0.61 \pm 0.05$ $\mathrm{mg} / \mathrm{mm} 3$ to $1.02 \pm 0.14 \mathrm{mg} / \mathrm{mm}^{3}$.

\section{Table 1.}

Morphological and structural-mechanical characteristics of wet composite CaAPGPs

\begin{tabular}{|c|c|c|}
\hline Gel particles & $\begin{array}{c}\text { Diameter, } \\
\mathrm{mm}\end{array}$ & $\begin{array}{c}\text { Density, } \\
\mathrm{mg} / \mathrm{mm}^{3}\end{array}$ \\
\hline $1 \% \mathrm{AP}+2 \% \mathrm{AA}$ & $4.28 \pm 0.25$ & $0.35 \pm 0.05$ \\
\hline $2 \% \mathrm{AP}+2 \% \mathrm{AA}$ & $3.80 \pm 0.25$ & $0.59 \pm 0.10$ \\
\hline $2 \% \mathrm{AP}+1 \% \mathrm{AA}$ & $4.03 \pm 0.20$ & $0.48 \pm 0.09$ \\
\hline $1 \% \mathrm{HS}+2 \% \mathrm{AA}$ & $3.47 \pm 0.08$ & $0.61 \pm 0.05$ \\
\hline $2 \% \mathrm{HS}+2 \% \mathrm{AA}$ & $3.27 \pm 0.15$ & $1.02 \pm 0.14$ \\
\hline $2 \% \mathrm{HS}+1 \% \mathrm{AA}$ & $3.50 \pm 0.15$ & $0.74 \pm 0.09$ \\
\hline
\end{tabular}

Table 2 shows the morphological and structuralmechanical (density) characteristics of dry CaAPGPs. It was found that dry CaAPGPs based on AP (Ca-AA-AP) have a diameter of $1.16 \pm 0.14-1.23 \pm 0.05 \mathrm{~mm}$, which was greater than the diameter of dry CaAPGPs based on HS (Ca-AA-HS) $(0.95 \pm 0.12-1.16 \pm 0.05 \mathrm{~mm})$. The density of dry CaAPGPs based on AP (Ca-AA-AP) with an increase in the concentration of AP in their composition from $1 \%$ to $2 \%$ increased by 1.7 times from $0.37 \pm 0.07 \mathrm{mg} / \mathrm{mm}^{3}$ to $0.63 \pm 0.05 \mathrm{mg} / \mathrm{mm}^{3}$. Dry composite CaAPGPs based on HS (Ca-AA-HS) were denser. With an increase in the HS concentration in their composition from $1 \%$ to $2 \%$, the degree of particle density increases by 2.2 - from $0.45 \pm 0.03 \mathrm{mg} / \mathrm{mm}^{3}$ to $0.97 \pm 0.19 \mathrm{mg} / \mathrm{mm}^{3}$.

Table 2.

Morphological and structural-mechanical characteristics of dry composite CaAPGPs

\begin{tabular}{|c|c|c|}
\hline Gel particles & $\begin{array}{c}\text { Diameter, } \\
\mathrm{mm}\end{array}$ & $\begin{array}{c}\text { Density, } \\
\mathrm{mg} / \mathrm{mm}^{3}\end{array}$ \\
\hline $1 \% \mathrm{AP}+2 \% \mathrm{AA}$ & $1.23 \pm 0.05$ & $0.37 \pm 0.07$ \\
\hline $2 \% \mathrm{AP}+2 \% \mathrm{AA}$ & $1.16 \pm 0.14$ & $0.63 \pm 0.05$ \\
\hline $2 \% \mathrm{AP}+1 \% \mathrm{AA}$ & $1.19 \pm 0.05$ & $0.46 \pm 0.08$ \\
\hline $1 \% \mathrm{HS}+2 \% \mathrm{AA}$ & $1.16 \pm 0.05$ & $0.45 \pm 0.03$ \\
\hline $2 \% \mathrm{HS}+2 \% \mathrm{AA}$ & $0.95 \pm 0.12$ & $0.97 \pm 0.19$ \\
\hline $2 \% \mathrm{HS}+1 \% \mathrm{AA}$ & $1.14 \pm 0.07$ & $0.69 \pm 0.12$ \\
\hline
\end{tabular}

The swelling and degradation of the obtained dry composite CaAPGPs in an artificial gastroenteric environment were studied.
It was found that CaAPGPs formed from 1\% AP and $2 \%$ AA degraded almost immediately in SIF. Whereas, CaAPGPs formed from $2 \%$ AP and $1 \%$ or $2 \%$ AA completely degraded in SCF after 1 hour of incubation in it (Fig. 1).

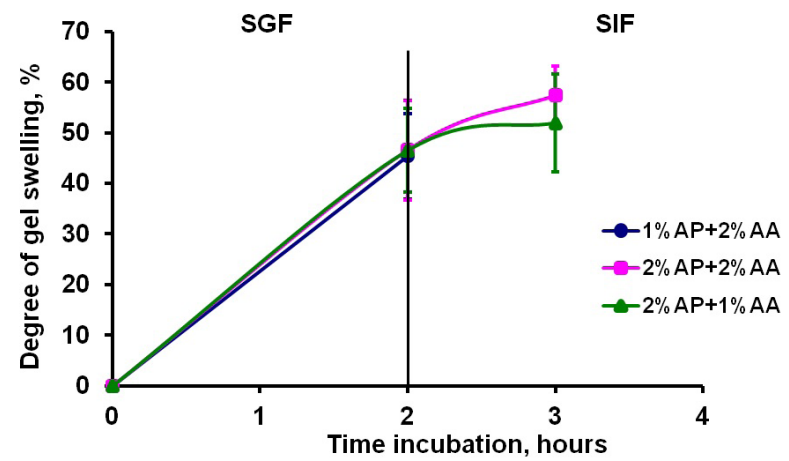

Fig. 1. Swelling and degradation of composite CaAPGPS formed on the basis of AP in an artificial GIT.

CaAPGPs formed from $1 \% \mathrm{HS}$ and $2 \% \mathrm{AA}$, and particles obtained from $2 \%$ HS and $1 \%$ AA, remained stable in SIF, and then completely degraded immediately upon entering in SCF. CaAPGPs, consisting of $2 \% \mathrm{HS}$ and $2 \% \mathrm{AA}$, dissolve in SCF after 1 hour of incubation in it. (Fig. 2).

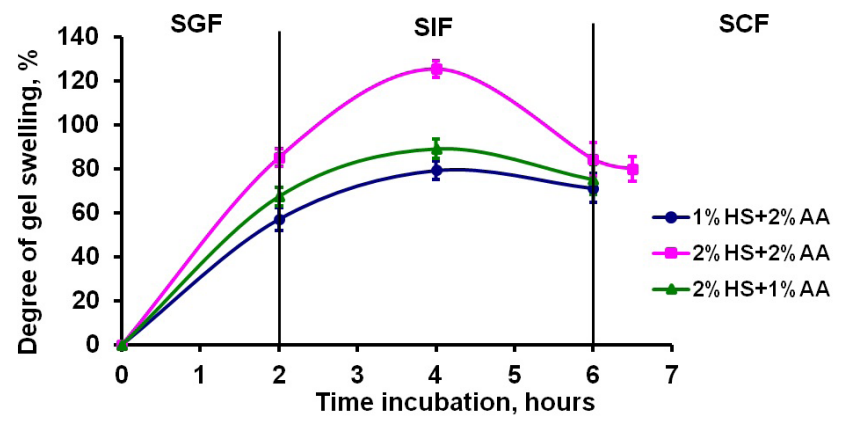

Fig. 2. Swelling and degradation of composite CaAPGPS formed on the basis of pectin HS in an artificial GIT.

Thus, our results indicate the promise of studying the obtained CaAPGPs in conditions of the artificial GIT as adsorbents of heavy metals that can adsorb heavy metals and remove them from the body.

\section{Competing Interest} interests.

The authors declare that they have no competing

*Corresponding author: Elena A. Mikhailova. Institute of Physiology of Komi Science Centre of the Ural Branch of the RAS, FRC Komi SC UB RAS. Syktyvkar, the Russian Federation. E-mail: elkina@physiol.komisc.ru 


\section{Sources of Funding}

The work was performed on the theme of research work (State registration number AAAA-A17-117012310147-8).

\section{References}

1. Ovodov YuS. [Current ideas about pectin substances]. Bioorg Khim. 2009; 35 (3): 293-310. [Article in Russian]. 2. Shubakov AA, Mikhailova EA, Prosheva VI, Belyy VA. Swelling and degradation of calcium-pectic gel particles made of pectins of Silene vulgaris and Lemna minor callus cultures at different concentrations of pectinase in an artificial colon environment. International Journal of Biomedicine. 2018; 8 (1): 60-64. doi: 10.21103 / Article8 (1)_OA10.

3. Minzanova ST, Mironov VF, Arkhipova DM, Khabibullina AV, Mironova LG, Zakirova YM, Milyukov VA. Biological activity and pharmacological application of pectic polysaccharides: A review. Polymers. 2018;10:1407. doi: 10.3390 / polym 10121407 .

4. Nie Y, Lin Q, Luo F. Effects of non-starch polysaccharides on inflammatory bowel disease. Int J Mol Sci. 2017;18:1372. doi: 10.3390 / ijms18071372.

5. Shubakov AA, Mikhailova EA. Production, properties and swelling of composite pectic-gel particles in an artificial gastric environment.International Journal of Biomedicine. 2021;11(2):173-176. doi: 10.21103 / Article11 (2) OA10.

6. Oliveira GF, Ferrari PC, Carvalho LQ, Evangelista RC.
Chitosan-pectin multiparticulate systems associated with enteric polymers for colonic drug delivery. Carbohydr Polym. 2010;82(3):1004-1009. doi: 10.1016/j.carbpol.2010.06.041

7. Günter EA, Popeyko OV, Belozerov VS, Martinson EA, Litvinets SG. Physicochemical and swelling properties of composite gel microparticles based on alginate and callus cultures pectins with low and high degrees of methylesterification. Int J Biol Macromol. 2020 Dec 1;164:863-870. doi: 10.1016/j.ijbiomac.2020.07.189.

8. Günter EA, Martynov VV, Belozerov VS, Martinson EA, Litvinets SG. Characterization and swelling properties of composite gel microparticles based on the pectin and $\kappa$-carrageenan. Int J Biol Macromol. 2020 Dec 1;164:22322239. doi: 10.1016/j.ijbiomac.2020.08.024.

9. Patova OA, Golovchenko VV, Vityazev FV, Burkov AA, Litvinets SG, Martinson EA, Belyi VA, Kuznetsov SN. Physicochemical and rheological properties of gelling pectin from Sosnowskyi's hogweed (Heracleum sosnowskyi) obtained using different pretreatment conditions. Food Hydrocolloids. 2017;65:77-86. doi: 10.1016/j.foodhyd.2016.10.042.

10. Sriamornsak P. Effect of calcium concentration, hardening agent and drying condition on release characteristics of oral proteins from calcium pectinate gel beads. Eur J Pharm Sci. 1999 Jul;8(3):221-7. doi: 10.1016/s0928-0987(99)00010-x.

11. Gebara C, Chaves KS, Ribeiro MCE, Souza FN, Grosso CRF, Gigante ML. Viability of Lactobacillus acidophilus La5 in pectin-whey protein microparticles during exposure to simulated gastrointestinal conditions. Food Res Int. 2013;51:872-878. doi: 10.1016/j.foodres.2013.02.008. 